\title{
QUALIDADE DA DIETA E FATORES DE RISCO PARA DOENÇAS CRÔNICAS
}

\author{
Caroline Taiane Thumé ${ }^{1 *} \&$ Fabiana Assmann Poll ${ }^{1}$
}

TRUMÉ, C,T,; \& POLL, F.A.;. Qualidade da dieta e fatores de risco para doenças crônicas. Perspectivas Online:

Biológicas \& Saúde. v. 8, n 26, p.31-41, 2018.

\section{RESUMO}

O presente estudo teve como objetivo verificar o Índice de Alimentação Saudável de servidores públicos e associar com possíveis fatores de risco para as Doenças Crônicas Não Transmissíveis (DCNT). Trata-se de uma pesquisa de delineamento transversal com funcionários públicos adultos. O consumo alimentar foi medido pelo método Recordatório Alimentar de 24 horas e a qualidade da dieta avaliada pelo Índice de Alimentação Saudável proposto por Mota et al. (2008). Os fatores de risco foram verificados através de um questionário com questões referentes ao estilo de vida, dados clínicos e nutricionais. Foram avaliados 28 indivíduos, $78,6 \%$ do sexo feminino, com idade média de
$38,68 \pm 11,48$ anos, sendo que $3,6 \%$ possuiu uma dieta de boa qualidade, $89,3 \%$ dieta precisando de melhorias e $7,1 \%$ dieta de má qualidade. O estado nutricional mais prevalente foi eutrofia (50\%) seguido de sobrepeso/obesidade $(42,9 \%)$. Não praticam atividade física $35,7 \%, 92,9 \%$ referiu não fumar, e $50 \%$ relataram ingerir bebida alcoólica. A presença de DCNT na família foi referida por $71,6 \%$ e $46,4 \%$ referem ter patologias atuais. Conclui-se que o padrão de qualidade da dieta que predominou foi o de precisando de melhorias, e que houve a presença de diversos fatores de risco para doenças crônicas nessa amostra.

Palavras-chave: Qualidade da dieta; Doenças crônicas não transmissíveis; Fatores de risco; Funcionários públicos. 


\begin{abstract}
This study aimed to verify the Healthy Eating Index of public servants and associate with possible risk factors for Chronic Noncommunicable Diseases (CNCDs). It is crosssectional research with adult civil servants. Food consumption was measured by the 24-hour Food Recall method and the quality of the diet evaluated by the Healthy Eating Index proposed by Mota et al. (2008). The risk factors were verified through a questionnaire with questions related to lifestyle, clinical and nutritional data. A total of 28 people were evaluated, $78.6 \%$ female, with a mean age of $38.68 \pm 11.48$ years, from

whom $3.6 \%$ has a good quality diet, $89.3 \%$ the diet is requiring improvements and $7,1 \%$ has a poor diet. The most prevalent nutritional status was eutrophia (50\%) followed by overweight/obesity (42.9\%). $35.7 \%$ do not practice physical activity, $92.9 \%$ reported not smoking, and 50\% reported ingesting alcohol. The presence of CNCDs in the family was reported by $71.6 \%$ and $46.4 \%$ reported having current pathologies. It was concluded that the diet quality standard that predominated was the one that needs improvements and that there were several risk factors for chronic diseases in this sample.
\end{abstract}

Keywords: Diet quality; Chronic Non-communicable Diseases; Risk factors; Public servants.

${ }^{1}$ Universidade de Santa Cruz do Sul - UNISC - Avenida Independência, 2293 - Universitário, Santa Cruz do Sul - RS, CEP:96815-900, Brasil.

(*) E-mail: carolthume94@ hotmail.com

Data de chegada: 14/11/2017 Aceito para publicação: 10/04/2018 


\section{INTRODUÇÃO}

Segundo a Organização Mundial da Saúde (OMS, 2005), as Doenças Crônicas Não Transmissíveis (DCNT) estão entre as principais causas de morte em quase todos os países, atingem principalmente aqueles de baixa e média renda, e geram piora na qualidade de vida, morte prematura e afetam economicamente a população em geral. De um total de 38 milhões de mortes ocorridas no mundo em 2012, 68\% foram atribuídas as DCNT (WHO, 2014). Igualmente no Brasil, em 2011, 68,3\% das mortes foram decorridas das DCNT, com relevância para as doenças cardiovasculares $(30,4 \%)$, as neoplasias $(16,4 \%)$, as doenças respiratórias (6\%) e o diabetes $(5,3 \%)$ (MALTA et al., 2014).

As DCNT não possuem causas nitidamente estabelecidas devido a sua etiologia múltipla (BRASIL, 2008): são o resultado de estilos de vida não saudáveis e desregrados (OMS, 2005). Os fatores de risco modificáveis relevantes são a hipertensão arterial, tabagismo, consumo excessivo de álcool, inatividade física, sobrepeso e obesidade, consumo inadequado de frutas e hortaliças e hiperglicemia (CAMPOS; NETO, 2009).

As transformações econômicas, políticas, sociais e culturais da sociedade atual provocam mudanças na forma de organizar a vida e no modo de viver da população. Como consequência disto, estas mudanças podem dificultar o acesso às condições de vida mais favoráveis à saúde, repercutindo diretamente nos padrões de adoecimento (BRASIL, 2008). Hoje, a condição determinante para a situação de saúde é a adoção de um estilo de vida saudável: apresentar estado nutricional, comportamento preventivo, controle do estresse e relacionamentos sociais satisfatórios para alcançar a longevidade com vitalidade (ALMEIDA; GUTIERREZ; MARQUES, 2012; SOUZA et al., 2016).

A qualidade da dieta tem consequências significativas na saúde das pessoas e os aspectos negativos da alimentação da população brasileira no final da primeira década do século XXI indicam a alta prioridade de políticas públicas de promoção da alimentação saudável (LEVY et al., 2012). Através da Pesquisa Nacional de Saúde de 2013, realizou-se um estudo sobre a prevalência do consumo de alimentos não saudáveis relacionados à DCNT no Brasil: estava presente com importante relevância o consumo de carne com excesso de gordura $(37,2 \%)$, leite integral $(60,6 \%)$, refrigerantes ou suco artificial $(23,4 \%)$ e doces $(21,7 \%)$, o que mostrou que a população brasileira apresentou alta prevalência de consumo de alimentos não saudáveis considerados fatores de risco para DCNT (CLARO et al., 2015).

Transformações benéficas no estilo de vida têm efeito direto sobre a saúde, em qualquer fase da vida ou condição preexistente de saúde (BARRETO et al., 2005). O seguimento de um padrão alimentar saudável rico em frutas e vegetais, legumes, grãos integrais, com foco na variedade de dieta, densidade de nutrientes e quantidade adequada de alimentos, e limitando a ingestão de energia a partir de gorduras saturadas, pode ser uma possível solução para os desafios atuais de prevenção e gerenciamento de obesidade e doenças cardiovasculares (ANDERSON, 2018).

Existem ferramentas para identificação da qualidade da dieta, e uma delas é o Índice de Alimentação Saudável Americano - Healthy Eating Index, que permite avaliar a qualidade global da dieta de indivíduos, e não simplesmente componentes isolados (KENNEDY et al., 1995). Para a sua utilização com a população brasileira, o Índice de Alimentação Saudável (IAS) foi adaptado com base no Guia Alimentar para a População Brasileira (MOTA et al., 2008).

Neste sentido, o presente estudo teve como objetivo verificar o Índice de Alimentação Saudável de indivíduos adultos, servidores públicos do município de Santa Cruz do Sul/RS, e associar com possíveis fatores de risco para as Doenças Crônicas Não Transmissíveis. 


\section{METODOLOGIA}

Trata-se de um estudo com delineamento transversal e abordagem qualitativa e quantitativa, realizado no período de julho a novembro de 2017 com funcionários públicos da Secretaria Municipal de Políticas Públicas do município de Santa Cruz do Sul/RS. O projeto de pesquisa foi aprovado pelo Comitê de Ética em Pesquisa da Universidade de Santa Cruz do Sul sob o parecer $n^{\circ}$ 2.167.757. O processo de amostragem foi feito por conveniência, incluíram-se na pesquisa funcionários públicos adultos de ambos os sexos, que aceitaram participar da pesquisa mediante a assinatura do termo de consentimento livre e esclarecido previamente à coleta de dados, como critérios de exclusão teve-se não ser funcionário público da Prefeitura Municipal de Santa Cruz do Sul, funcionários com idade inferior a 18 e superior a 59 anos, e não assinar o termo de consentimento livre e esclarecido.

A qualidade da dieta foi avaliada pelo IAS brasileiro proposto por Mota et al. (2008), realizado mediante o inquérito do Recordatório Alimentar de 24 horas (R24h) dos indivíduos. Os fatores de risco para DCNT foram verificados através de um questionário elaborados pelas pesquisadoras, com questões fechadas e abertas referentes ao estilo de vida, dados clínicos e nutricionais. Tanto o R24h como o questionário foram auto preenchidos pelos indivíduos em seus domicílios, após a entrega e explicação da pesquisadora em seus locais de trabalho. Deu-se o prazo de três dias para devolução após o preenchimento.

O formulário do R24h foi fornecido e explicado ao indivíduo para autopreenchimento, com os seguintes itens: refeição, local, alimento/bebidas, quantidade e observações. Foi disponibilizado um material referente às medidas caseiras para facilitar o seu preenchimento (VITOLO, 2008). Solicitou-se o registro de dois dias, em duas semanas distintas e consecutivas, porém no mesmo dia da semana. Já o questionário para identificação de fatores de risco da DCNT de estilo de vida, clínicos e nutricionais constou das seguintes questões fechadas: sexo, atividade física, tabagismo e consumo de álcool. E questões abertas foram referentes à presença de DCNT na família, patologias atuais, peso auto referido e altura auto referida.

Os resultados do questionário foram confrontados com o IAS apresentado por cada indivíduo através do R24h com o objetivo de analisar os hábitos de vida daqueles que apresentam boa qualidade da dieta, precisando de melhorias ou má qualidade. Os R24h foram analisados através do software Dietbox ${ }^{\circledR}$. Todos os alimentos foram convertidos para gramas e mililitros, separados por grupos alimentares e transformados em porções, conforme valor energético equivalente a uma porção proposta pela Pirâmide Alimentar Adaptada, como apresentado na Tabela 1 (PHILIPPI et al., 1999). Além disso, considerou-se a variedade de alimentos, e a quantidade de gordura total, gordura saturada e colesterol disponibilizada no software após o cálculo dos R24h.

Tabela 1: Valor energético equivalente a uma porção dos grupos alimentares da Pirâmide Alimentar Adaptada. Fonte: Adaptado de Philippi et al. (1999)

\begin{tabular}{cc}
\hline Grupos alimentares da Pirâmide Alimentar Adaptada & Valor energético (kcal) \\
\hline Cereais, pães, raízes e tubérculos & 150 \\
Hortaliças & 15 \\
Frutas & 35 \\
Leguminosas e oleaginosas & 55 \\
Carnes e ovos & 190 \\
Leite e produtos lácteos & 120 \\
Óleos e gorduras & 73 \\
Açúcares e doces & 110 \\
\hline
\end{tabular}


Para calcular a pontuação total de todas as refeições realizadas de acordo com o IAS, foram somadas as porções de cada grupo. O IAS contém 12 itens, cada item pode pontuar de 0 a 10 , totalizando num máximo de 120 pontos. A distribuição da pontuação do IAS está demonstrada na Tabela 2. Os indivíduos que ingeriram a quantidade de porções recomendadas pela Pirâmide Alimentar Adaptada pontuaram nota máxima, aqueles que não ingeriram nenhuma porção ganharam nota mínima e aqueles que ingeriram entre o número de porções recomendadas e nenhuma porção, foi feito cálculo proporcional (MOTA et al., 2008).

Tabela 2: Componentes do Índice de Alimentação Saudável e o seu critério de pontuação. Fonte: Mota et al. (2008)

\begin{tabular}{lccc}
\hline & Pontuação & $\begin{array}{c}\text { Pontuação máxima } \\
\text { de } \mathbf{1 0}\end{array}$ & $\begin{array}{c}\text { Pontuação mínima } \\
\text { de 0 }\end{array}$ \\
\hline $\begin{array}{l}\text { Grupo dos cereais, pães, } \\
\text { tubérculos e raízes }\end{array}$ & 0 a 10 & $5-9$ porções & 0 porções \\
Grupo dos vegetais & 0 a 10 & $4-5$ porções & 0 porções \\
Grupo das frutas & 0 a 10 & $3-5$ porções & 0 porções \\
Grupo das leguminosas & 0 a 10 & 1 porção & 0 porções \\
Grupo das carnes & 0 a 10 & $1-2$ porções & 0 porções \\
Grupo dos laticínios & 0 a 10 & 3 porções & 0 porções \\
Grupo dos óleos e gorduras & 0 a 10 & $1-2$ porções & 0 porções \\
Grupo dos açúcares e doces & 0 a 10 & $1-2$ porções & 0 porções \\
Gordura total $(\%)$ & 0 a 10 & $\leq 30$ & $\geq 45$ \\
Gordura saturada $(\%)$ & 0 a 10 & $<10$ & $\geq 15$ \\
Colesterol alimentar $(\mathbf{m g})$ & 0 a 10 & $\leq 300$ & $\geq 450$ \\
Variedade & 0 a 10 & $\geq 8$ diferentes & $\leq 3$ diferentes \\
& & itens/dia & itens/dia \\
\hline
\end{tabular}

Os dois R24h foram analisados separadamente e calculados a pontuação à parte. Em seguida foi feito a média dessas pontuações para obtenção da pontuação final. A qualidade da dieta foi determinada segundo a soma dos pontos de todos os 12 componentes, obtendo a pontuação final e a respectiva classificação: pontuação final < 71 pontos foram consideradas de "má qualidade", com pontuação entre 71 e 100 pontos "precisando de melhorias" e >100 pontos "boa qualidade" (MOTA et al., 2008).

Para avaliação do estado nutricional utilizou-se a classificação proposta pelo Índice de Massa Corporal (IMC) (OMS, 2000), sendo que foram unidas as classificações "sobrepeso/obesidade" para fins de análise dos resultados. Os fatores de risco para DCNT foram analisados descritivamente.

Os resultados foram analisados através de estatística descritiva, e de associações entre a classificação da qualidade da dieta obtida pelo IAS e demais variáveis do estilo de vida e classificação estado nutricional no programa Statistical Package for Social Sciences (SPSS) versão 20.0. Em virtude da classificação "precisando de melhorias" ter tido um percentual bem maior que os demais nessa amostra, optamos por analisar as características intra grupo.

\section{RESULTADOS E DISCUSSÃO}

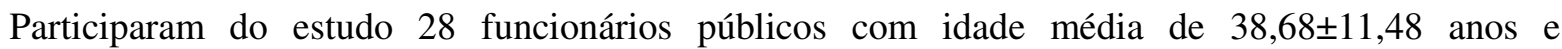
predominância do sexo feminino $(78,6 \%)$. A metade deste público $(50 \%)$ apresentou estado nutricional de 
eutrofia, 42,9\% sobrepeso/obesidade, e 7,1\% magreza, ou seja, valores mais positivos do que os mostrados na Vigitel de 2016, cuja prevalência de excesso de peso somada a obesidade foi de 72,7\% (BRASIL, 2017).

A prática de atividade física foi referida de 1 a 3 vezes/semana e de 4 a 7 vezes/semana por 32,1\% dos funcionários em cada frequência, e aqueles que não praticam, foram totalizados em $35,7 \%$. A maioria não fuma $(92,9 \%)$, porém a metade da amostra relatou ingerir bebida alcoólica (50\%), sem considerar a frequência. Também, 71,4\% relataram que há presença de DCNT na família e 46,4\% referem ter patologias

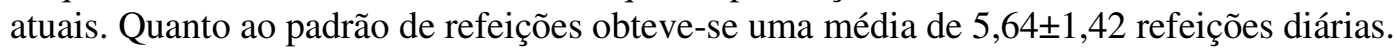

Observou-se que a maioria dos participantes do estudo (89,3\%) possui uma dieta que necessita de melhorias, segundo o IAS, conforme apresentado na Tabela 3.

Tabela 3: Caracterização da qualidade da dieta segundo o Índice de Alimentação Saudável (IAS) (n=28)

\begin{tabular}{lc}
\hline Classificação da dieta & Frequência \\
\hline Boa qualidade & $1(3,6 \%)$ \\
Precisando de melhorias & $25(89,3 \%)$ \\
Má qualidade & $2(7,1 \%)$ \\
\hline
\end{tabular}

Semelhante ao resultado do presente estudo, Felippe et al. (2011) avaliaram o IAS envolvendo 100 indivíduos adultos, em dois grupos diferentes: um grupo exposto a um programa de reeducação alimentar e o outro sem nenhuma orientação nutricional - em ambos os grupos, a maior parte dos participantes foi classificada como precisando melhorar a qualidade da dieta: $70,4 \%$ e $71,4 \%$ respectivamente. Este estudo concluiu que indivíduos que recebem orientação nutricional apresentam uma dieta de melhor qualidade quando comparados àqueles sem acompanhamento nutricional.

Já a pesquisa realizada por Lima et al. (2013), utilizando o IAS, com 747 beneficiários adultos do Programa Bolsa Família, maioria feminina $(91,4 \%)$, também mostrou que o público está precisando de melhorias na dieta, sendo que 52,9\% foram classificados com dieta inadequada, $45,7 \%$ com dieta que necessita de modificações e apenas 1,3\% com dieta adequada. Both, Franz e Busnello (2012) avaliaram a qualidade da dieta de frequentadores de academia e, do mesmo modo, a maioria deste público apresentou dietas precisando de melhorias (57\%). Nespeca e Cyrillo (2010) em pesquisa com funcionários públicos $(\mathrm{n}=276)$ mostrou que a média do IAS foi de 68,1 pontos, classificando a dieta dos participantes como merecedora de melhorias.

Esta característica quanto à necessidade de melhora na dieta foi comum nos estudos e pode ser observada na alimentação da população brasileira, e justificada pela diminuição do consumo de frutas, verduras e legumes e o aumento no consumo de alimentos com altas quantidades de açúcar simples, de gordura e de sal. Um dos motivos desta transição alimentar é o aumento da diversidade de produtos industrializados e o aumento da disseminação dos mesmos (BRASIL, 2009).

Os resultados dos indivíduos, que compõem a amostra que obteve a classificação "precisando de melhorias" constam na Tabela 4. 
Tabela 4: Caracterização da população com qualidade da dieta "precisando de melhorias" ( $\mathrm{n}=25)$

\begin{tabular}{|c|c|c|}
\hline Variável & Média $\pm D P$ & Frequência \\
\hline Idade (anos) & $38,60 \pm 11,61$ & \\
\hline \multicolumn{3}{|l|}{ Sexo } \\
\hline Feminino & & $19(76,0 \%)$ \\
\hline Masculino & & $6(24,0 \%)$ \\
\hline \multicolumn{3}{|l|}{ Estado Nutricional } \\
\hline Magreza & & $2(8,0 \%)$ \\
\hline Eutrofia & & $12(48,0 \%)$ \\
\hline Sobrepeso/obesidade & & $11(44,0 \%)$ \\
\hline \multicolumn{3}{|l|}{ Prática de Atividade Física } \\
\hline Nunca & & $8(32,0 \%)$ \\
\hline $1-3$ vezes por semana & & $8(32,0 \%)$ \\
\hline 4-7 vezes por semana & & $9(36,0 \%)$ \\
\hline \multicolumn{3}{|l|}{ Ingestão de bebida alcóolica } \\
\hline Não & & $13(52,0 \%)$ \\
\hline Sim & & $12(48,0 \%)$ \\
\hline \multicolumn{3}{|l|}{ Tabagismo } \\
\hline Sim & & $2(8,0 \%)$ \\
\hline Não & & $23(92,0 \%)$ \\
\hline \multicolumn{3}{|l|}{ DCNT familiar } \\
\hline Sim & & $18(72,0 \%)$ \\
\hline Não & & $7(28,0 \%)$ \\
\hline \multicolumn{3}{|l|}{ Apresenta patologias atualmente } \\
\hline Sim & & $12(48,0 \%)$ \\
\hline Não & & $13(52,0 \%)$ \\
\hline \multicolumn{3}{|c|}{ Tipo de Patologia apresentada $(n=12)$} \\
\hline Hipertensão Arterial Sistêmica & & $1(8,3 \%)$ \\
\hline Diabetes Mellitus & & $1(8,3 \%)$ \\
\hline Doenças respiratórias & & $3(25,0 \%)$ \\
\hline Doença cardiovascular & & $2(16,7 \%)$ \\
\hline Dislipidemia & & $1(8,3 \%)$ \\
\hline Outro tipo de patologia & & $4(33,3 \%)$ \\
\hline Refeições diárias & $5,72 \pm 1,40$ & \\
\hline
\end{tabular}

No presente estudo, o estado nutricional sobrepeso/obesidade apresentou prevalência de 42,9\% na amostra total, o que demonstra ser menor do que em outros estudos, tais como de Nespeca e Cyrillo (2011) que avaliaram o estado nutricional de 276 funcionários públicos da Universidade de São Paulo e destes, $62,8 \%$ encontravam-se em sobrepeso/obesidade; de Sousa et al. (2015) que verificaram o estado nutricional de 52 servidores públicos que participavam do "Projeto Qualidade de Vida no trabalho - Ginástica no CEU", em Minas Gerais, e destes, 53,8\% foram diagnosticados com excesso de peso; e por último Moreira, Greco e Chaoubah (2016), que classificaram o estado nutricional de 579 servidores públicos técnico administrativos da Universidade Federal de Juiz de Fora e encontraram 60,4\% de sobrepeso/obesidade.

A frequência desse estado nutricional, porém, é similar a dados brasileiros que mostram que cerca de $40 \%$ dos indivíduos adultos do país encontram-se em estado de excesso de peso, não havendo diferença significativa entre homens e mulheres (BRASIL, 2008). Isso se deve a conjectura global atual derivada dos padrões de vida inadequados provenientes do consumo de alimentos de alta densidade calórica, ingestão 
inadequada de micronutrientes, alimentos ultraprocessados, e sedentarismo, com a diminuição das atividades físicas e do lazer ativo, em detrimento da televisão e dos jogos eletrônicos (SOARES et al., 2013-2014).

A média de refeições diárias do público com classificação da dieta "precisando de melhorias" ficou em 5,72 $\pm 1,40$, e, portanto segue a recomendação mínima diária orientada pelo Guia Alimentar para a População Brasileira de três refeições principais intercaladas com lanches (BRASIL, 2008).

Quanto à prática de exercício físico, apenas $36 \%$ praticam na maioria dos dias da semana (4 a 7x/semana) conforme orienta a OMS (2004). Segundo a Estratégia Global para Dieta, Atividade Física e Saúde da OMS (2004), recomendam-se 30 minutos de atividade física regular de moderada intensidade, na maioria dos dias da semana, para apresentar resultados na saúde, tais como redução do risco de doenças cardiovasculares e diabetes, câncer de cólon e câncer de mama.

Um estudo, realizado por Lee et al. (2012), mostrou que as DCNT podem ser agravadas pela falta de atividade física. Foi estimado que a inatividade física causou a mortalidade de $6 \%$ por doenças coronarianas, $7 \%$ por diabetes tipo $2,10 \%$ por câncer de mama e $10 \%$ por câncer de cólon, acarretando em $9 \%$ de mortalidade prematura, ou mais de 5,3 milhões dos 57 milhões de óbitos ocorridos em todo o mundo em 2008.

Fazendo uma relação da prática de atividade física com a qualidade da dieta, Costa et al. (2012) realizou uma pesquisa com 182 mulheres usuárias de um programa de atividade física regular "Academia da Cidade", e o desfecho foi: $90,6 \%$ desta população apresentou dieta com necessidade de modificações, concluindo que são importantes a realização de estratégias educacionais voltadas à nutrição, na efetivação de uma vida saudável, visto que a maioria destas eram mais novas e com excesso de peso.

O tabagismo foi pouco prevalente na amostra estudada, visto que a grande maioria não faz uso do tabaco (92\%), o que é positivo para a saúde destas pessoas. O hábito de fumar é uma ameaça à saúde, pois compromete a qualidade e a expectativa de vida - os fumantes tem uma expectativa de vida muito inferior aos não-fumantes. (NICITA-MAURO, 2008).

Em relação ao consumo de bebida alcoólica, $48 \%$ do público com dieta precisando de melhorias referiram fazer uso. Yeomans (2004) sugere em seu estudo que a ingestão de bebida alcoólica tem um efeito estimulador de apetite em curto prazo, além de que bebidas alcoólicas são calóricas e as pessoas não reduzem a ingestão de outras fontes de energia quando ingerem bebida alcoólica, indicando que o álcool possa ser um fator de risco para o ganho de peso, ocasionando prejuízos para a saúde das pessoas.

Com relação a presença de doenças crônicas, $72 \%$ afirmaram ter familiares de primeiro grau com DCNT e $48 \%$ tem alguma patologia atualmente. A herança genética também é um fator de risco para a ocorrência de DCNT (BRASIL, 2008). Estes dados deveriam ser um indicativo de cuidados na alimentação para prevenção de futuras complicações na saúde, o que foi demonstrado em parte, pelo resultado do IAS na categoria que aponta que a dieta ainda precisa de melhorias. O estudo realizado por Carvalho e Rocha (2011) também mostrou esta preocupação: foi aplicado um questionário de frequência alimentar em 150 adultos residentes em área rural da cidade de Ibatiba (ES) e os resultados encontrados foram alto consumo de gordura animal e açúcares e baixo consumo de frutas onde concluíram que o hábito alimentar da população estudada pode vir a incrementar a médio ou longo prazo a prevalência e/ou ocorrência de DCNT.

Para promover saúde e prevenir as DCNT, o Brasil conta com uma ampla política que engloba uma série de ações em articulação intersetorial, educação em saúde, monitoramento de doenças e de fatores de risco, e fornecimento de atenção à saúde centrada em dietas saudáveis, prática regular de exercício físico, redução do tabagismo e do consumo de álcool (SCHMIDT et al., 2011). Todavia foi notável ao longo da 
pesquisa que se faz necessária maior conscientização quanto à adoção de hábitos saudáveis para a prevenção de doenças e que as pessoas precisam priorizar atitudes mais adequadas de alimentação e estilo de vida.

Foram encontradas limitações no presente estudo, as quais o tamanho amostral se limitou pequeno, o que dificultou a representatividade da população-alvo estudada. Outra limitação potencial foi que o R24h e o questionário utilizados na metodologia foram auto preenchidos, o que pode favorecer a omissão de informações. Porém, pesquisas que avaliam hábitos e consumo alimentar, mesmo que não na modalidade de serem "auto referidos", constantemente enfrentam essas limitações, já que dependem do relato dos indivíduos sobre sua rotina de vida e alimentar.

Apesar disso, a relevância de pesquisas nesse âmbito, tais como o presente estudo, são indiscutíveis, pois a alimentação junto ao estilo de vida, formam um conjunto de fatores de risco modificáveis na gênese das DCNT, que tem tido prevalência cada vez maior nas estatísticas de saúde, assim possibilitando as pessoas a identificação e modificação de padrões de vida, visando promoção de saúde.

Vale salientar um ponto importante sobre o IAS, a variância da pontuação do grupo "precisando de melhorias" foi grande (71-100 pontos), e que a perda de pontos em dois ou três componentes da ferramenta já classifica a qualidade da dieta como precisando de melhorias. Também, este método não considerou a qualidade do alimento ingerido pelos participantes, como exemplo, pode-se citar se os cereais são integrais ou refinados, se a refeição contém todos nutrientes previstos para atingir as necessidades nutricionais individuais, entre outros. Além disto, não avalia a quantidade máxima de porções consumida, visto que, quando o indivíduo atinge o número mínimo de porções recomendadas, já pontua nota máxima no componente.

\section{CONCLUSÕES}

Concluiu-se que no presente estudo a qualidade da dieta para a grande maioria da amostra avaliada foi classificada como "precisando de melhorias". Quanto aos aspectos clínicos, nutricionais e estilo de vida, obteve-se que aproximadamente um terço não pratica exercícios físicos, $42,9 \%$ estão em sobrepeso/obesidade, $46 \%$ referem presença de patologias atuais e $71,4 \%$ DCNT em familiares, que a presença de tabagismo foi baixa, mas o consumo de álcool (sem considerar a frequência) foi relatado por $50 \%$ dos indivíduos. Resultados semelhantes foram obtidos quando se analisou somente a população classificada como "precisando de melhorias" na dieta.

Esses achados corroboram com os objetivos da pesquisa e diversos estudos realizados com esse público e tema. Percebe-se a presença de fatores de risco cardiovasculares relacionados ao estilo de vida e dados clínicos, assim como aspectos nutricionais inadequados para manutenção de uma vida saudável. O que nos remete considerar que são necessárias estratégias de prevenção e promoção da saúde, para estimular modificações efetivas de comportamentos, que favorecem a prevenção de problemas de saúde futuros, tais como a manifestação de DCNT.

\section{REFERÊNCIAS}

ALMEIDA, M. A. B.; GUTIERREZ, G. L.; MARQUES, R. Qualidade de vida: definição, conceitos e interfaces com outras áreas de pesquisa. São Paulo: Escola de Artes, Ciências e Humanidades - EACH/USP, 2012.

ANDERSON, C. A. M. Dietary Patterns to Reduce Weight and Optimize Cardiovascular Health: Persuasive Evidence for Promoting Multiple, Healthful Approaches. Circulation, fev. 2018.

BARRETO, S. M. et al. Análise da Estratégia Global para Alimentação, Atividade Física e Saúde, da Organização Mundial da Saúde. Epidemiologia e Serviços de Saúde, v. 14, n. 1, p. 41-68, jan./mar. 2005. 
BOTH, M.; FRANZ, L. B. B.; BUSNELLO, M. B. Índice de qualidade da dieta de frequentadores de academia. Rev. Contexto e Saúde, Ijuí, v. 12, n. 23, p.2-8, jul./dez. 2012.

BRASIL. Ministério da Saúde. Secretaria de Vigilância à Saúde. Secretaria de Atenção à Saúde. Diretrizes e recomendações para o cuidado integral de doenças crônicas não-transmissíveis: promoção da saúde, vigilância, prevenção e assistência. Brasília: Ministério da Saúde, 2008.

BRASIL. Ministério da Saúde. Secretaria de Atenção à Saúde. Guia alimentar para a população brasileira: promovendo a alimentação saudável. Brasília: Ministério da Saúde, 2008.

BRASIL. Ministério da Educação. Secretaria de Educação Básica. Módulo 10: Alimentação e nutrição no Brasil. Brasília: Universidade de Brasília, 2009.

BRASIL. Ministério da Saúde. Secretaria de Vigilância em Saúde. Vigitel Brasil 2016: vigilância de fatores de risco e proteção para doenças crônicas por inquérito telefônico: estimativas sobre frequência e distribuição sociodemográfica de fatores de risco e proteção para doenças crônicas nas capitais dos 26 estados brasileiros e no Distrito Federal em 2016. Brasília: Ministério da Saúde, 2017.

CAMPOS, M. O.; NETO, J. F. R. Doenças Crônicas não Transmissíveis: fatores de risco e repercussão na qualidade de vida. Revista Baiana de Saúde Pública, v. 33, n.4, p.561-581, out./dez. 2009.

CARVALHO, E. O.; ROCHA, E. F. Consumo alimentar de população adulta residente em área rural da cidade de Ibatiba (ES, Brasil). Ciênc. saúde coletiva, Rio de Janeiro, v. 16, n. 1, p. 179-185, jan. 2011.

CLARO, R. M. et al. Consumo de alimentos não saudáveis relacionados a doenças crônicas não transmissíveis no Brasil: Pesquisa Nacional de Saúde, 2013. Epidemiol. Serv. Saúde, Brasília, v. 24, n. 2, p. 257-265, jun. 2015.

COSTA, D. et al. Índice de qualidade da dieta de mulheres usuárias de um programa de atividade física regular "Academia da Cidade", Aracajú, SE. Rev. Nutr., Campinas, v. 25, n. 6, p. 731-741, dez. 2012.

FELIPPE, F. et al. Qualidade da dieta de indivíduos expostos e não expostos a um programa de reeducação alimentar. Rev. Nutr., Campinas, v. 24, n. 6, p. 833-844, dez. 2011.

KENNEDY, E. T. et al. The Healthy Eating Index: design and applications. Journal of the American Dietetic Association. v. 95, n. 10, p. 1103-1108, out. 1995.

LEE I. M. et al. Effect of physical inactivity on major non-communicable diseases worldwide: an analysis of burden of disease and life expectancy. Lancet. v. 380, p. 219-229, jul. 2012.

LEVY R. B. et al. Distribuição regional e socioeconômica da disponibilidade domiciliar de alimentos no Brasil em 2008-2009. Rev. Saúde Pública, São Paulo, v. 46, n. 1, p. 06-15, fev. 2012.

LIMA, F. E. L. et al. Programa Bolsa-Família: qualidade da dieta de população adulta do município de Curitiba, PR. Rev. bras. epidemiol., São Paulo, v. 16, n. 1, p. 58-67, mar. 2013.

MALTA, D. C. et al. Mortalidade por doenças crônicas não transmissíveis no Brasil e suas regiões, 2000 a 2011. Epidemiol. Serv. Saúde, v. 23, n. 4, p. 599-608, 2014.

MOREIRA, L. B. F., GRECO, R. M., CHAOUBAH, A. Estado nutricional dos servidores técnicos administrativos em educação de uma universidade pública associado aos hábitos alimentares. Braspen Journal, Juiz de Fora, v. 31, n. 3, p. 213-218, 2016.

MOREIRA, P. R. S. et al. Análise crítica da qualidade da dieta da população brasileira segundo o Índice de Alimentação Saudável: uma revisão sistemática. Ciênc. saúde coletiva, Rio de Janeiro, v. 20, n. 12, p. 39073923, dez. 2015.

MOTA, J. F. et al. Adaptação do índice de alimentação saudável ao guia alimentar da população brasileira. Rev. Nutr., Campinas, v. 21, n. 5, p. 545-552, out. 2008. 
NESPECA, M.; CYRILLO, D. C. Avaliação da qualidade da dieta por meio do índice de alimentação saudável de funcionários de uma universidade pública. Nutrire: rev. Soc. Bras. Alim., São Paulo, v. 35, n. 2 , p. 81-90, ago. 2010.

NESPECA, M.; CYRILLO, D. C. Qualidade de vida no trabalho de funcionários públicos: papel da nutrição e da qualidade de vida. Acta Scientiarum. Health Sciences, Maringá, v. 33, n. 2, p. 187-195, jul./dez. 2011.

NICITA-MAURO, V. et al. Smoking, aging and the centenarians. Exp. Gerontol., v. 43, n. 2, p. 95-101, fev. 2008.

ORGANIZAÇÃO MUNDIAL DA SAÚDE. Prevenção de doenças crônicas: um investimento vital. Genebra: OMS, 2005.

PHILIPPI, S. T. et al. Pirâmide Alimentar Adaptada: guia para escolha dos alimentos. Rev. Nutr., Campinas, v. 12, n. 1, p. 65-80, jan./abr. 1999.

SCHMIDT, M. I. et al. Doenças crônicas não transmissíveis no Brasil: carga e desafios atuais. Lancet, v. 4, p. 61-74, mai. 2011.

SOARES, L. R. et al. A transição da desnutrição para a obesidade. Brazilian Journal of Surgery and Clinical Research, v. 5, n. 1, p. 64-68, dez. 2013/fev. 2014.

SOUSA, T. M. et al. Adesão de Servidores Públicos a Programa de Qualidade de Vida e Intervenção Nutricional. O Mundo da Saúde, São Paulo, v. 39, n. 1, p. 92-101, 2015.

SOUZA, M. A. H.et al. Perfil do estilo de vida de longevos. Rev. bras. geriatr. gerontol., Rio de Janeiro, v. 19, n. 5, p. 819-826, out. 2016.

VITOLO, M. R. Nutrição: da gestação ao envelhecimento. Rio de Janeiro: Ed. Rubio, 2008.

WORLD HEALTH ORGANIZATION. Obesity: preventing and managing the global epidemic. Report of a WHO Consultation. WHO Technical Report Series 894. Geneva: World Health Organization, 2000.

WORLD HEALTH ORGANIZATION. Global strategy on diet, physical activity and health. Geneva: World Health Organization, 2004.

WORLD HEALTH ORGANIZATION. Global status report on noncommunicable diseases 2014. Geneva, 2014.

YEOMANS, M. R. Effects of alcohol on food and energy intake in human subjects: evidence for passive and active over-consumption of energy. British Journal of Nutrition, v. 92, n. 1, p. S31-S34, 2004. 